\title{
Effect of Joints' Configuration Change on the Effective Mass of the Robot
}

\author{
Abdel-Nasser Sharkawy a, ${ }^{\text {** }}$ \\ ${ }^{a}$ Mechatronics Engineering, Mechanical Engineering Department, Faculty of Engineering, South Valley University, \\ Qena 83523, Egypt. \\ 1 abdelnassersharkawy@eng.svu.edu.eg \\ * Corresponding Author
}

\section{ARTICLE INFO}

\section{Article history}

Received January 06, 2022

Revised January 24, 2022

Accepted February 18, 2022

Keywords

Robot Effective Mass;

Joint Configuration;

UR10e;

KUKA LBR iiwa 7 R800;

Mathematical Analysis

\begin{abstract}
The effective mass of the robot is considered of great significance in enhancing the safety of human-robot collaboration. In this paper, the effective mass of the robot is investigated using different joint configurations. This investigation is executed in two steps. In the first step, the position of each joint of the robot is changing alone, whereas the positions of the other joints of the robot are fixed, and then the effective mass is determined. In the second step, the positions of all joints of the robot are changing together, and the effective mass of the robot is determined. From this process, the relation between the effective mass of the robot and the joint configurations can be presented. This analysis is implemented in MATLAB and uses two collaborative robots; the first one is the UR10e robot which is a 6-DOF robot, and the second one is the KUKA LBR iiwa 7 R800 robot, which is a 7-DOF robot. The results from this simulation prove that the change in any joint position of the robot except the first and the last joint affect the effective mass of the robot. In addition, the change in all joints' positions of the robot affects the effective mass. Effective mass can thus be considered as one of the criteria in optimizing the robot kinematics and configuration.
\end{abstract}

This is an open-access article under the CC-BY-SA license.

\section{Introduction}

Starting from rest, the effective mass of the robot is defined as [1] the inverse of the magnitude of the component of the linear acceleration along the direction $u$ that results from a unit force applied along this direction $u$. Sometimes, it is also called reflected mass such [2]-[6].

Safety is the most necessary stage during human-robot collaboration since the operator's proximity to the robot may lead to the possibility of injuries. Researchers sought to implement collision avoidance techniques as in [7]-[12]. Furthermore, collision detection techniques were implemented to improve the safety system in human-robot interaction if the level of collision avoidance fails. These techniques were whether model-based [13]-[15] or data-based approaches [16]-[23].

The effective mass of robots is also considered of great significance in improving the safety of human-robot cooperation. Gang Chen and his group [24] designed a strategy of minimizing the contact force based on the effective mass for the space flexible manipulator. This approach decreases the 
contact force via deriving the collision direction and the pre-configuration. S. D. Lee et al. [6] designed a collision model to evaluate the collisions' safety for any spatial manipulator. This collision model relates the design parameters to collision safety by adopting the robot's effective mass and manipulability. Mavrakis et al. [25] presented how the safety of the post-grasp motion could be considered in the phase of the pre-grasp approach. Therefore, the selected grasp is optimal in terms of applying the minimum impact forces if the collision occurs during the desired post-grasp manipulation. They built on the methods of the augmented robot-object dynamics models and the effective mass. In [26], a virtual sensor approach was proposed to compute the collision peak force and the pressure that results from the human-robot collision at a given time using an analytical contact model. The collision safety was evaluated using the conventional finite element simulation for particular collision conditions with the given parameters, which include the effective mass, the collision velocity and direction, and the impactor shape. In [27], the workspace's effective mass distribution was investigated. In addition, an experiment was conducted for deriving the effective mass by observing the impulse received by an object, and implications were drawn on human safety and the robot efficiency in human-robot interaction.

In [28], Na and his group statistically analyzed the effective mass and inertia in an expected workspace for representing the inertial properties of the haptic device.

In a previous paper [29], the mathematical analysis of the effective mass of the robot and its ellipsoid was presented. The effective mass of the robot was investigated with the collisions affecting the robot end-effector, and the results from this investigation proved that the collision force depends on the effective mass of the robot. Furthermore, the effective mass was analysed using different robot configurations and different end-effector positions using 2-DOF and 3-DOF planar robots. The results from this analysis proved that the effective mass of the robot is related to the robot configurations and end-effector position.

From this discussion, we observe that further investigation and analysis are necessary for the effective mass of the robot by using different joint configurations.

The main contribution of this paper is presented as follows:

- The relation between the effective mass of the robot and the joints' configurations are investigated and analyzed in detail.

- This investigation is presented by changing the position of each joint of the robot alone, but the other joints of the robot are fixed, and then the effective mass of the robot is determined. The second step is that all positions of the joints of the robot are changing together, and then the effective mass of the robot is determined.

- This analysis is very crucial to show how each joint's position affects the effective mass of the robot and then how all joints' positions influence the effective mass.

This analysis is executed in MATLAB and using the collaborative robots; UR10e robot (6-DOF) and KUKA LBR iiwa 7 R800 robot (7-DOF).

The outline of this paper is presented as follows. Section 2 shows how the effective mass of the robot can be calculated. In section 3, the investigation of the effective mass by changing the joints' configurations of the robot is presented in detail. Section 4 summarizes the main important points presented in this paper and provides future works.

\section{Effective Mass Equation}

The effective mass of the robot is determined along the direction vector $u$ by the following equation [1]:

$$
m^{-1}=u^{T}\left(J(q) M(q)^{-1} J^{T}(q)\right) u=u^{T} H^{-1} u
$$

Therefore, 


$$
m=\frac{1}{u^{T} H^{-1} u}
$$

where, $J(q)$ is the Jacobian matrix of the manipulator, $M(q)$ is the inertia matrix of the manipulator, and $u$ is the direction vector describing the direction of the impact force applied to the robot endeffector.

This equation demonstrates that the effective mass of the robot is affected by its configuration and the collision direction.

\section{Effective Mass Analysis with Different Joint Configurations}

In this section, an investigation of the effective mass of the robot is presented using different joint configurations. In the first step, the position of each joint of the robot is changing alone, whereas other joints are fixed, and then the effective mass is determined. The second step is that the positions of all the joints of the manipulator are changing together, and then the effective mass of the robot is determined. This process is presented in Fig. 1.

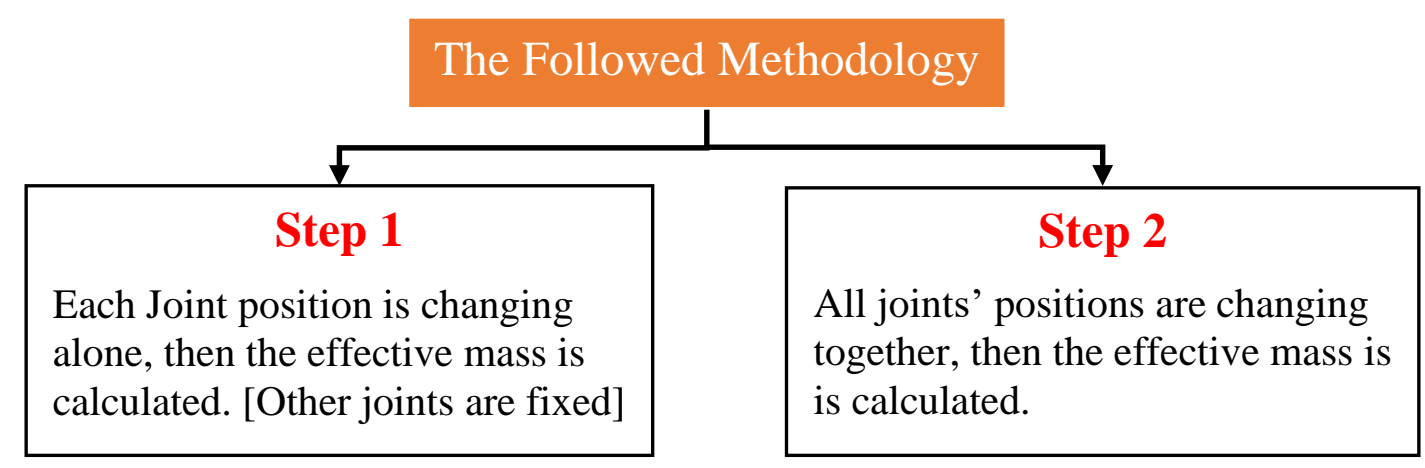

Fig. 1. The followed methodology in changing the joints' positions of the manipulator.

The analysis and investigation are carried out using two collaborative robots: UR10e robot (6DOF) and KUKA LBR iiwa 7 R800 robot (7-DOF), and it is executed in MATLAB. These types of robots are shown in Fig. 2.

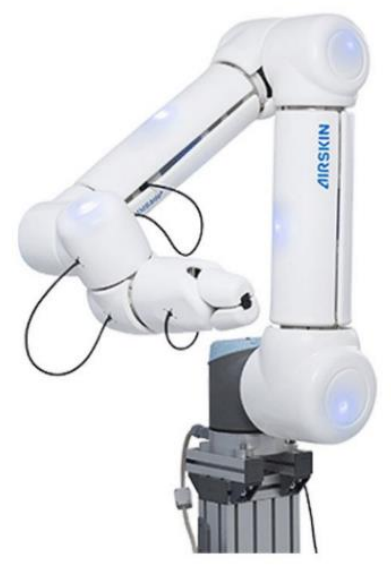

(a)

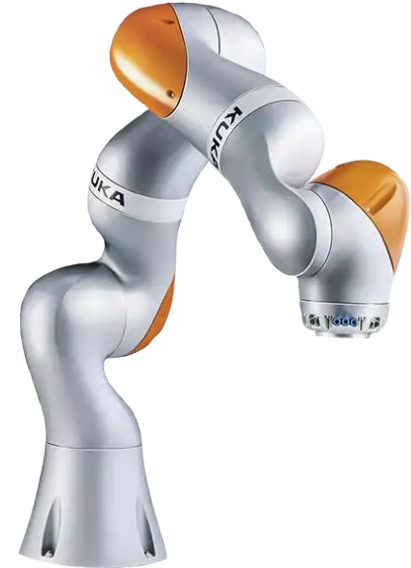

(b)

Fig. 2. The two collaborative robots. (a) UR10e robot (6-DOF) and (b) KUKA LBR iiwa 7 R800 robot (7DOF). The Figures are taken from ref. [30], [31]. 


\subsection{Investigation Using UR10e Robot (6-DOF)}

\subsubsection{The Position of Each Joint is Changing Alone}

In this step, a simulation in MATLAB is carried out by changing the position of each joint of the manipulator alone, but the other joints of the manipulator are fixed. The workspace of each joint is taken into consideration. The motion range of each joint of the UR10e robot (6-DOF) is presented in Table 1. They followed protocol, and steps for executing this simulation are presented in Table 2 (step 1 to step 6).

Table 1. The motion range of each joint of UR10e robot.

\begin{tabular}{lcccccc}
\hline Joint & 1 & 2 & 3 & 4 & 5 & 6 \\
\hline Range Motion (rad) & \pm 6.28 & \pm 6.28 & \pm 2.62 & +0.6 to -3.75 & \pm 4.7 & \pm 6.28 \\
\hline
\end{tabular}

Table 2. The followed protocol/steps for changing the joints' positions of UR10e robot.

\begin{tabular}{ccccccc}
\hline & \multicolumn{7}{c}{ Joint position (rad) } \\
\cline { 2 - 7 } Step & 1 & 2 & 3 & 4 & 5 & 6 \\
\hline Step 1 & {$[-5,5]$} & 3 & 1.5 & -2.5 & 3 & 3 \\
Step 2 & 3 & {$[-5,5]$} & 1.5 & -2.5 & 3 & 3 \\
\hline Step 3 & 3 & 3 & {$[-2,2]$} & -2.5 & 3 & 3 \\
\hline Step 4 & 3 & 3 & 1.5 & {$[-3.5,0.5]$} & 3 & 3 \\
Step 5 & 3 & 3 & 1.5 & -2.5 & {$[-4,4]$} & 3 \\
\hline Step 6 & 3 & 3 & 1.5 & -2.5 & 3 & {$[-5,5]$} \\
\hline Step 7 & {$[-5,5]$} & {$[-5,5]$} & {$[-2,2]$} & {$[-3.5,0.5]$} & {$[-4,4]$} & {$[-5,5]$} \\
\hline
\end{tabular}

The obtained results of this simulation are presented in Fig. 3. The results presented in Fig. 3 show that when the position of joint 1 is changing alone, or the position of joint 6 is changing alone, no change happens to the effective mass of the robot. When the position of joint 2 or joint 3 or joint 4 or joint 5 is changing alone, the effective mass is affected, and some decrease and increase happens to it. From these results, we can conclude that only the position of joints $2,3,4$, or 5 has an effect on the effective mass of the robot. It should be noted that the effective mass of the robot has a different value when the position of joint $1 \in[-5,2]$ rad and when the position of joint $1 \in[3,5] \mathrm{rad}$. This happens because the joints 2 to 6 are fixed in a position in the interval $[-5,2]$, and these joints are fixed in another position in the interval $[3,5]$.

\subsubsection{The Positions of all Joints are Changing Together}

In this step, a simulation is executed by changing the positions of all the joints of the UR10e robot simultaneously (see step 7 in Table 2). The results from this simulation are presented in Fig. 4.

The results obtained in Fig. 4 shows that changing the positions of all joints of the UR10e robot together affect the effective mass of the robot, and some increase and decrease happens to it.

From the results obtained in this section (3.1), we conclude that with the UR10e robot, all joints' positions affect the effective mass of the robot except the position of joint 1 and joint 6 . 

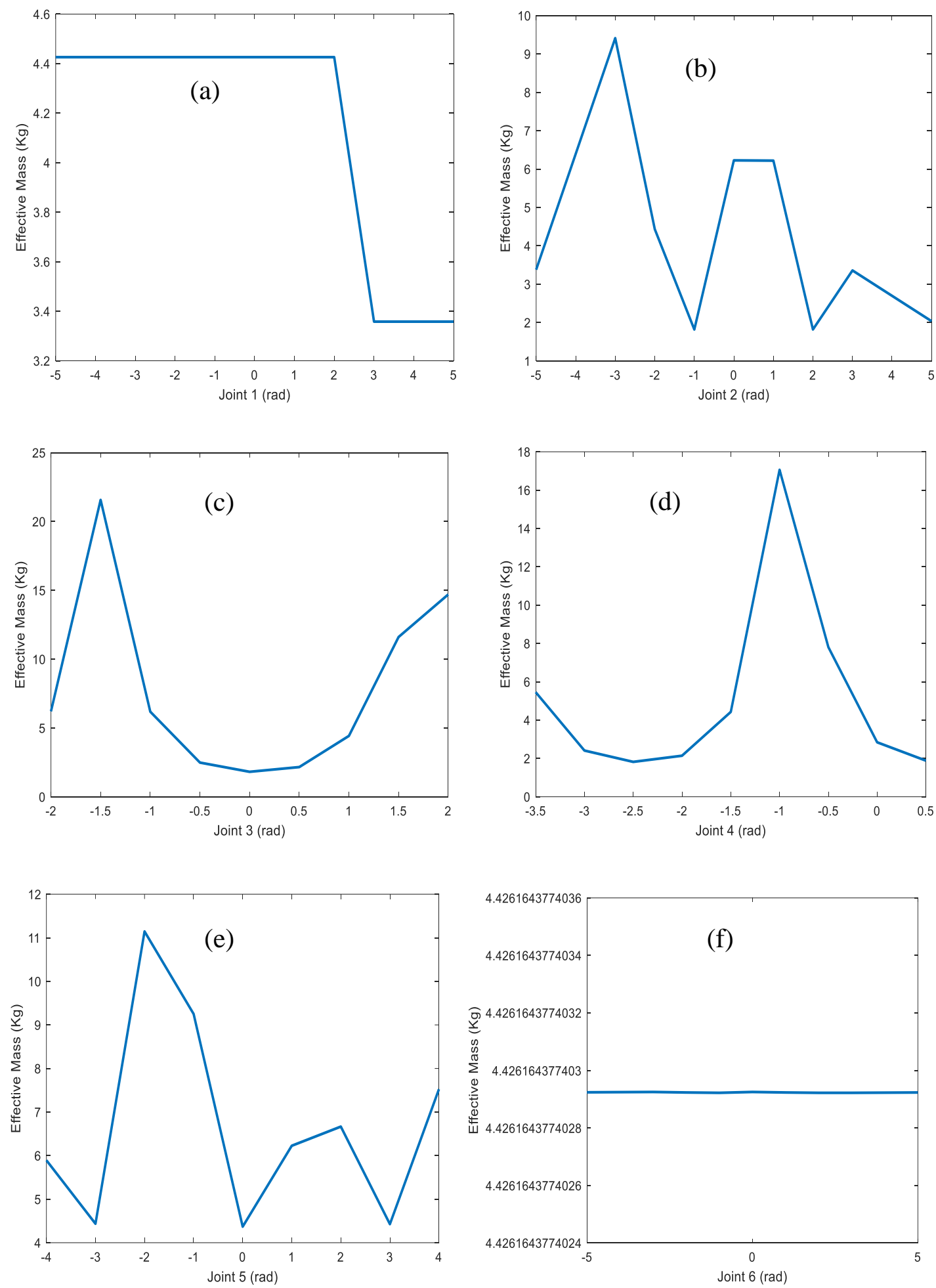

Fig. 3. The obtained effective mass of the robot by changing the position of each joint of UR10e robot alone. 


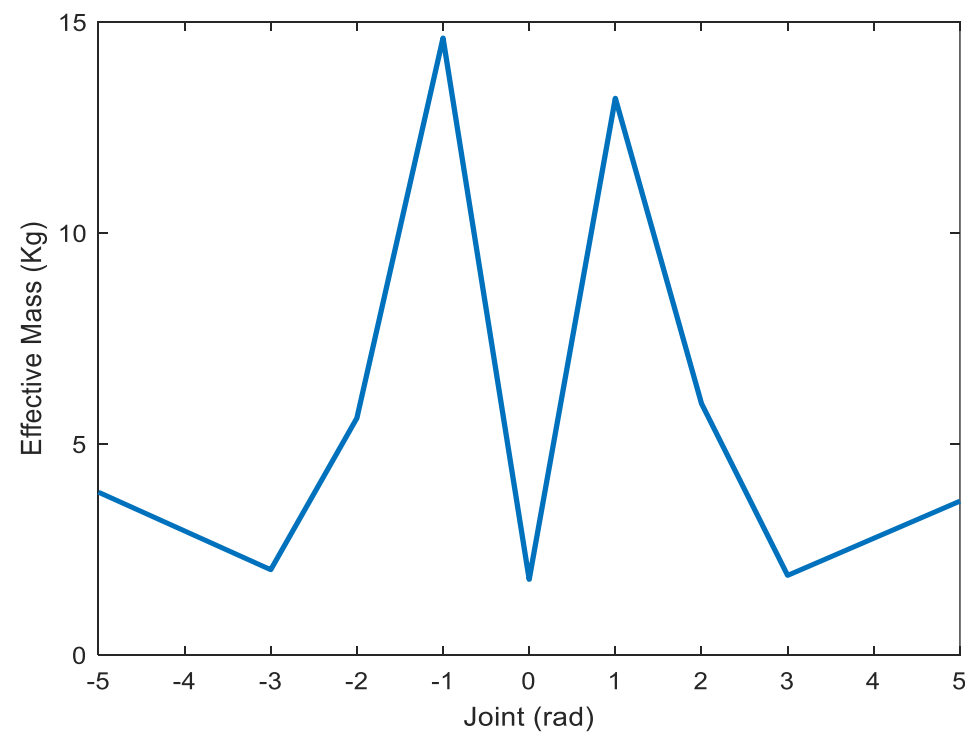

Fig. 4. The obtained effective mass by changing the positions of all joints of UR10e robot together.

\subsection{Investigation Using KUKA LBR iiwa 7 R800 Robot (7-DOF)}

\subsubsection{The Position of Each Joint is Changing Alone}

The same protocol done in section 3.1 is repeated here in this section. In the first step, the position of each joint of the KUKA LBR iiwa 7 R800 robot is changing alone, whereas the positions of other joints are fixed. The motion range of each joint of the robot is considered and presented in Table 3. The followed protocol, in this case, is presented in Table 4.

Table 3. The motion range of each joint of KUKA LBR iiwa 7 R800 robot [32].

\begin{tabular}{cccccccc}
\hline Joint & 1 & 2 & 3 & 4 & 5 & 6 & 7 \\
$\begin{array}{c}\text { Range Motion } \\
(\text { rad) }\end{array}$ & \pm 2.97 & \pm 2.1 & \pm 2.97 & \pm 2.1 & \pm 2.97 & \pm 2.1 & \pm 3.05 \\
\hline
\end{tabular}

Table 4. The followed protocol/steps for changing the joints' positions of the KUKA LBR iiwa 7 R800 robot.

\begin{tabular}{cccccccc}
\hline & \multicolumn{7}{c}{ Joint position (rad) } \\
\cline { 2 - 7 } Step & 1 & 2 & 3 & 4 & 5 & 6 & 7 \\
\hline Step 1 & {$[-2.5,2.5]$} & -1.5 & -2 & -1.5 & -2 & -1.5 & -2 \\
\hline Step 2 & -2 & {$[-2,2]$} & -2 & -1.5 & -2 & -1.5 & -2 \\
\hline Step 3 & -2 & -1.5 & {$[-2.5,2.5]$} & -1.5 & -2 & -1.5 & -2 \\
\hline Step 4 & -2 & -1.5 & -2 & {$[-2,2]$} & -2 & -1.5 & -2 \\
\hline Step 5 & -2 & -1.5 & -2 & -1.5 & {$[-2.5,2.5]$} & -1.5 & -2 \\
\hline Step 6 & -2 & -1.5 & -2 & -1.5 & -2 & {$[-2,2]$} & -2 \\
\hline Step 7 & -2 & -1.5 & -2 & -1.5 & -2 & -1.5 & {$[-2.9,2.9]$} \\
\hline Step 8 & {$[-2,2]$} & {$[-1.5,1.5]$} & {$[-2,2]$} & {$[-1.5,1.5]$} & {$[-2,2]$} & {$[-1.5,1.5]$} & {$[-2,2]$} \\
\hline
\end{tabular}



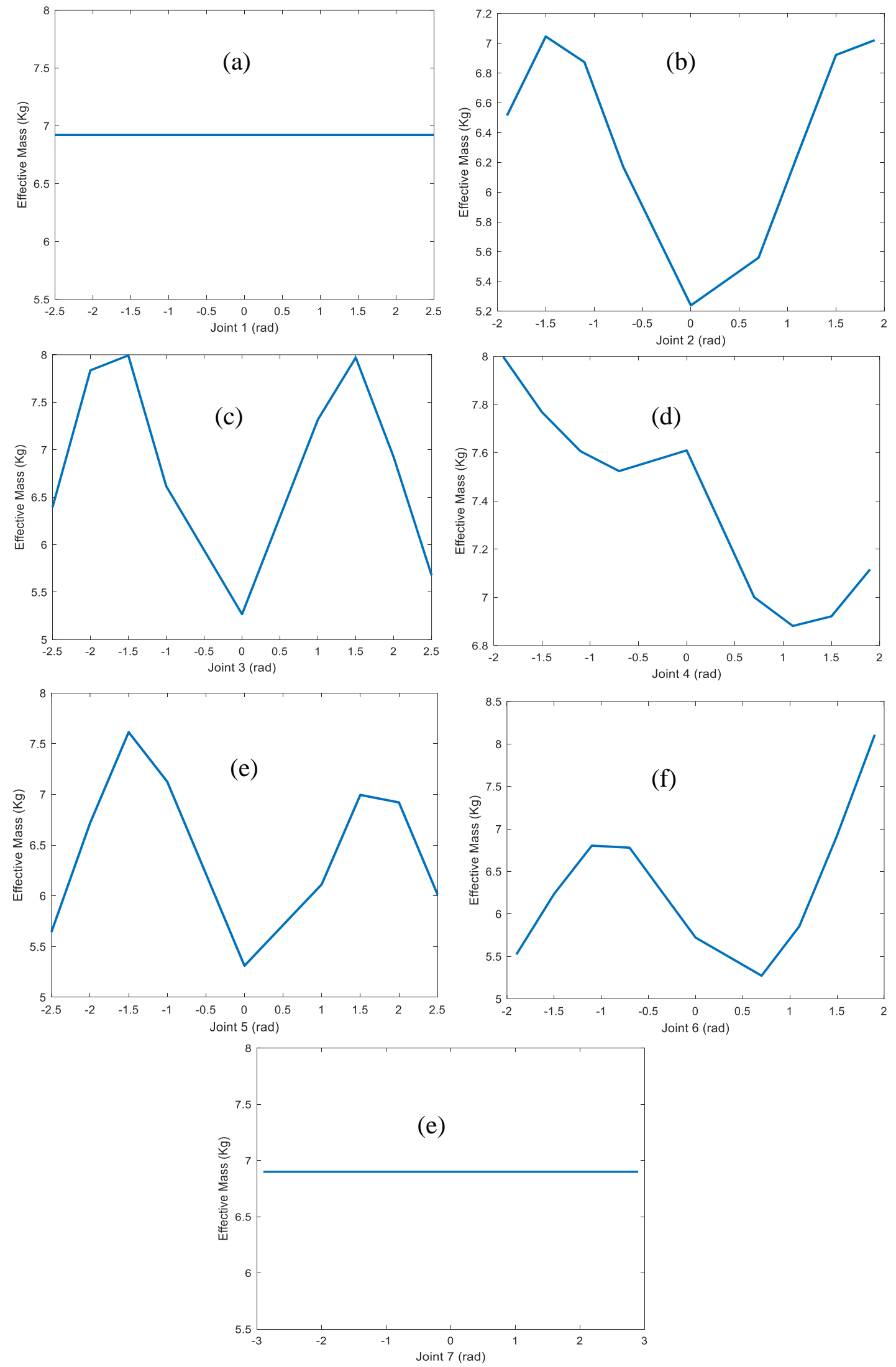

Fig. 5. The effective mass of the robot resulted by changing the position of each joint of KUKA LBR robot alone. 
The results obtained from this implemented simulation and protocol are shown in Fig. 5. These results present that the effective mass of the robot is affected by the change in the position of joints 2 , $3,4,5$, or 6 . The change in the position of joint 1 or joint 7 has no effect on the effective mass of the robot. These results support the ones obtained in subsection 3.1.1 with the UR10e robot.

\subsubsection{The Positions of all Joints are Changing Together}

In this step, the positions of all joints of the KUKA LBR iiwa 7 R800 robot are changing together (step 8 in Table 4). The results from this simulation are presented in Fig. 6. These results show that the change in all joints of the robot affects the effective mass of the robot. These results support the results obtained in subsection 3.1.2 with the UR10e robot.

From the results obtained in this section (3.2), we can conclude that with the KUKA LBR iiwa 7 R800 robot, the positions of all joints affect the effective mass of the robot except the position of joint 1 and joint 7.

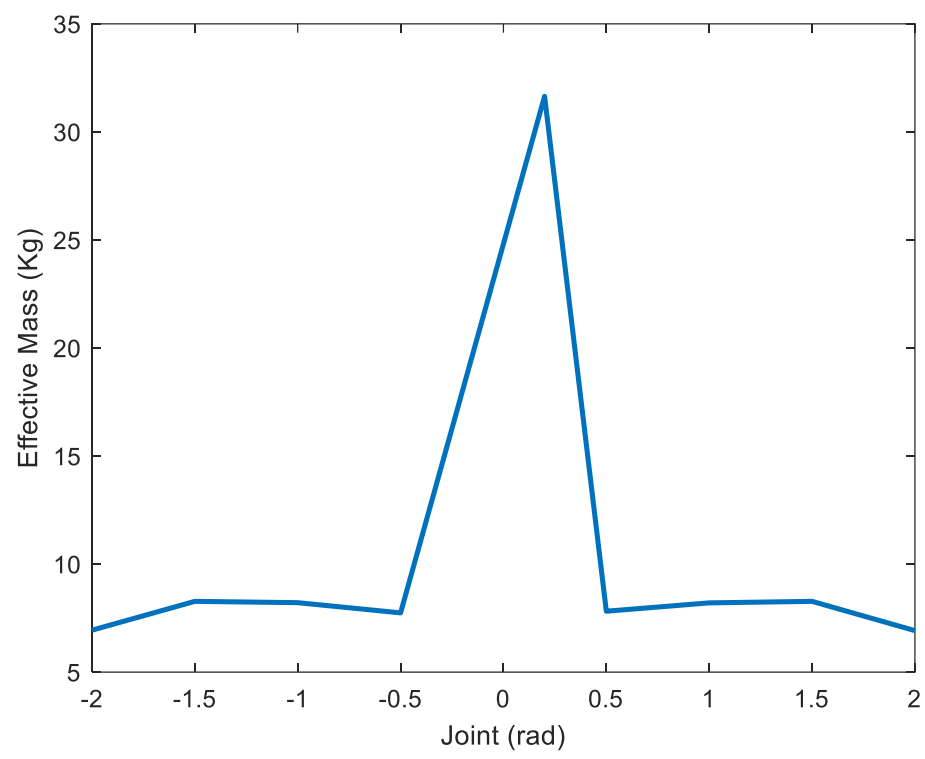

Fig. 6. The obtained effective mass by changing the positions of all joints of KUKA LBR iiwa 7 R800 robot together.

In final, the results from the simulation work presented in this section prove that for both the UR10e robot and KUKA LBR iiwa 7 R800 robot, 1) the change in the position of the first or the last joint of the robot has no effect on the effective mass of the robot, 2) the change in any other joint position affect the effective mass of the robot, and 3) the change of the positions of all joints of the robot affect the effective mass.

\section{Conclusions}

This paper investigates the relationship between the effective mass of the robot and the joints' configurations. A simulation study is executed in MATLAB by changing the position of each joint of the robot alone, whereas the positions of other joints of the robot are fixed, and then the effective mass is calculated. The results from this simulation show that the change in the position of the first or the last joint of the robot has no effect on the effective mass of the robot. The change in any other joint affects the effective mass. The second simulation is changing the positions of all the joints of the manipulator together. The results from this simulation prove that the effective mass of the robot is affected by the change in all joints together. Both simulations are carried out using two collaborative robots: the UR10e robot (6-DOF) and the KUKA LBR iiwa 7 R800 robot (7-DOF). Future work can consider the relationship between the position of the robot end-effector and the effective mass of the robot using the two robots: UR10e robot and KUKA LBR iiwa 7 R800 robots. In addition, experimental work can be conducted, and then the effective mass of the robot is presented. 
Conflict of interest: The author declares that there is no conflict of interest.

\section{References}

[1] O. Khatib, "Inertial Properties in Robotic Manipulation: An Object-Level Framework," Int. J. Rob. Res., vol. 14, no. 1, pp. 19-36, 1995, https://doi.org/10.1177/027836499501400103.

[2] S. Haddadin and E. Croft, "Physical Human-Robot Interaction," Springer Handbook of Robotics, Springer, 2016, pp. 1835-1874, https://doi.org/10.1007/978-3-319-32552-1_69.

[3] N. Mansfeld, M. Hamad, M. Becker, A. G. Marin, and S. Haddadin, "Safety map: A unified representation for biomechanics impact data and robot instantaneous dynamic properties," IEEE Robot. Autom. Lett., vol. 3, no. 3, pp. 1880-1887, 2018. http://dx.doi.org/10.1109/LRA.2018.2801477.

[4] N. Lucci, B. Lacevic, A. M. Zanchettin, and P. Rocco, "Combining speed and separation monitoring with power and force limiting for safe collaborative robotics applications," IEEE Robot. Autom. Lett., vol. 5, no. 4, pp. 6121-6128, 2020, https://doi.org/10.1109/LRA.2020.3010211.

[5] S. Haddadin, "Physical Safety in Robotics," Formal Modeling and Verification of Cyber-Physical Systems: 1st International Summer School on Methods and Tools for the Design of Digital Systems, R. Drechsler and U. Kühne, Eds. Bremen, Germany: Springer, 2015, pp. 249-271. https://doi.org/10.1007/978-3-658-09994-7_9.

[6] S. D. Lee, B. S. Kim, and J. B. Song, "Human-robot collision model with effective mass and manipulability for design of a spatial manipulator," Adv. Robot., vol. 27, no. 3, pp. 189-198, 2013. https://doi.org/10.1080/01691864.2012.754076.

[7] F. Flacco, T. Kroger, A. De Luca, and O. Khatib, "A Depth Space Approach to Human-Robot Collision Avoidance," 2012 IEEE International Conference on Robotics and Automation, 2012, pp. 338-345. https://doi.org/10.1109/ICRA.2012.6225245.

[8] B. Schmidt and L. Wang, "Contact-less and Programming-less Human-Robot Collaboration," Forty Sixth CIRP Conference on Manufacturing Systems 2013, 2013, vol. 7, pp. 545-550. https://doi.org/10.1016/j.procir.2013.06.030.

[9] F. D. Anton, S. Anton, and T. Borangiu, "Human-Robot Natural Interaction with Collision Avoidance in Manufacturing Operations," Studies in Computational Intelligence, pp. 375-388, 2013, https://doi.org/10.1007/978-3-642-35852-4_24.

[10] M. Kitaoka, A. Yamashita, and T. Kaneko, "Obstacle Avoidance and Path Planning Using Color Information for a Biped Robot Equipped with a Stereo Camera System," Proceedings of the 4th Asia International Symposium on Mechatronics, 2010, pp. 38-43, https://doi.org/10.1109/IROS.2003.1250741.

[11] S. Lenser and M. Veloso, "Visual Sonar: Fast Obstacle Avoidance Using Monocular Vision," in Proceedings 2003 IEEE/RSJ International Conference on Intelligent Robots and Systems (IROS 2003), 2003, https://doi.org/10.1109/IROS.2003.1250741.

[12] T. L. Lam, H. W. Yip, H. Qian, and Y. Xu, "Collision Avoidance of Industrial Robot Arms using an Invisible Sensitive Skin,” 2012 IEEE/RSJ International Conference on Intelligent Robots and Systems, 2012, pp. 4542-4543, https://doi.org/10.1109/IROS.2012.6386294.

[13] S. Haddadin, A. Albu-sch, A. De Luca, and G. Hirzinger, "Collision Detection and Reaction: A Contribution to Safe Physical Human-Robot Interaction," 2008 IEEE/RSJ International Conference on Intelligent Robots and Systems, 2008, pp. 3356-3363, https://doi.org/10.1109/IROS.2008.4650764.

[14] C. Cho, J. Kim, S. Lee, and J. Song, "Collision detection and reaction on 7 DOF service robot arm using residual observer," J. Mech. Sci. Technol., vol. 26, no. 4, pp. 1197-1203, 2012, https://doi.org/10.1007/s12206-012-0230-0.

[15] S. Morinaga and K. Kosuge, "Collision Detection System for Manipulator Based on Adaptive Impedance Control Law," Proceedings of the 2003 IEEE International Conference on Robotics \&Automation, 2003, pp. 1080-1085, https://doi.org/10.1109/ROBOT.2003.1241736.

[16] F. Dimeas, L. D. Avendano-valencia, and N. Aspragathos, "Human - Robot collision detection and identification based on fuzzy and time series modelling," Robotica, vol. 33, no. 9, pp. 1886-1898, 2015, https://doi.org/10.1017/S0263574714001143. 
[17] S. Lu, J. H. Chung, and S. A. Velinsky, "Human-Robot Collision Detection and Identification Based on Wrist and Base Force / Torque Sensors," Proceedings of the 2005 IEEE International Conference on Robotics and Automation, 2005, pp. 3796-3801, https://doi.org/10.1109/ROBOT.2005.1570699.

[18] A.-N. Sharkawy and N. Aspragathos, "Human-Robot Collision Detection Based on Neural Networks," Int. J. Mech. Eng. Robot. Res., vol. 7, no. 2, pp. 150-157, 2018, https://doi.org/10.18178/ijmerr.7.2.150157.

[19] A.-N. Sharkawy, P. N. Koustoumpardis, and N. Aspragathos, "Manipulator Collision Detection and Collided Link Identification based on Neural Networks," Advances in Service and Industrial Robotics. RAAD 2018. Mechanisms and Machine Science, A. Nikos, K. Panagiotis, and M. Vassilis, Eds. Springer, Cham, 2018, pp. 3-12, https://doi.org/10.1007/978-3-030-00232-9_1.

[20] A. N. Sharkawy, P. N. Koustoumpardis, and N. Aspragathos, "Neural Network Design for Manipulator Collision Detection Based only on the Joint Position Sensors," Robotica, vol. 38, no. 10, pp. 17371755, 2020, https://doi.org/10.1017/S0263574719000985.

[21] A. N. Sharkawy, P. N. Koustoumpardis, and N. Aspragathos, "Human-robot collisions detection for safe human-robot interaction using one multi-input-output neural network," Soft Comput., vol. 24, no. 9, pp. 6687-6719, 2020, https://doi.org/10.1007/s00500-019-04306-7.

[22] A. N. Sharkawy and A. A. Mostfa, "Neural networks' design and training for safe human-robot cooperation," J. King Saud Univ. - Eng. Sci., pp. 1-15, 2021, https://doi.org/10.1016/j.jksues.2021.02.004.

[23] A.-N. Sharkawy, "Intelligent Control and Impedance Adjustment for Efficient Human-Robot Cooperation," University of Patras, 2020, http://dx.doi.org/10.12681/eadd/47954.

[24] G. Chen, D. Liu, Y. Wang, and Q. Jia, "Contact Force Minimization for Space Flexible Manipulators Based on Effective Mass," J. Guid. Dyn. Eng. Notes, vol. 42, no. 8, pp. 1870-1877, 2019, https://doi.org/10.2514/1.G003987.

[25] N. Mavrakis, A. M. G. E., and R. Stolkin, "Safe robotic grasping: Minimum impact-force grasp selection," IEEE International Conference on Intelligent Robots and Systems, 2017, pp. 4034-4041, https://doi.org/10.1109/IROS.2017.8206258.

[26] H. Shin, S. Kim, K. Seo, and S. Rhim, "A virtual pressure and force sensor for safety evaluation in collaboration robot application," Sensors (Switzerland), vol. 19, no. 19, pp. 1-11, 2019, https://doi.org/10.3390/s19194328.

[27] R. J. Kirschner, N. Mansfeld, G. G. Pena, S. Abdolshah, and S. Haddadin, "Notion on the correct use of the robot effective mass in the safety context and comments on ISO/TS 15066," ISR $2021-2021$ IEEE International Conference on Intelligence and Safety for Robotics, 2021, pp. 6-9, https://doi.org/10.1109/ISR50024.2021.9419495.

[28] Y. Na, J. Won, and J. Park, "Statistical Indices for Inertial Properties of Haptic Devices," The 7th International Conference on Ubiquitous Robots and Ambient Intelligence (URAI 2010) Statistical, 2010, pp. 1-4, http://dyros.snu.ac.kr/paper/URAI2010_YoonhoNa.pdf

[29] A. N. Sharkawy, "An investigation on the effective mass of the robot: Dependence on the end-effector position," Eng. Trans., vol. 69, no. 3, pp. 293-313, 2021, http://dx.doi.org/10.24423/EngTrans.1329.20210826.

[30] “AIRSKIN safe collision sensor for UR10.” [Online]. Available: https://cobots.ie/product/airskin-safecollision-sensor-for-ur10/.

[31] "KUKA Collaborative Robot Series, KUKA LBR IIWA 7 R800." [Online]. Available: https://www.robots.com/robots/lbr-iiwa-7-r800.

[32] KUKA Roboter GmbH, KUKA iiwa Lightweight Robot, LBR iiwa 7 R800, LBR iiwa 14 R820, Spez LBR i. D-86165 Augsburg, 2015. https://www.oir.caltech.edu/twiki_oir/pub/Palomar/ZTF/KUKARoboticArmMaterial/Spez_LBR_iiwa _en.pdf 